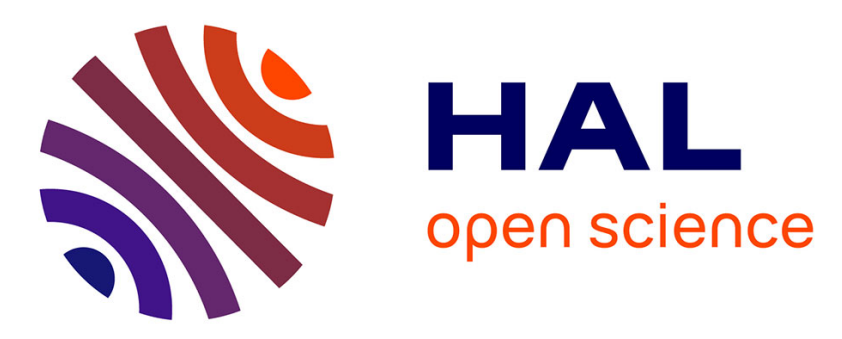

\title{
Efficient Media Streaming with Collaborative Terminals for the Smart City Environment
}

Jordi Mongay Battala, Piotr Krawiec, Constandinos Mavromoustakis, George

Mastorakis, Naveen Kumar Chilamkurti, Daniel Negru, Joachim

Bruneau-Queyreix, Eugen Borcoci

\section{To cite this version:}

Jordi Mongay Battala, Piotr Krawiec, Constandinos Mavromoustakis, George Mastorakis, Naveen Kumar Chilamkurti, et al.. Efficient Media Streaming with Collaborative Terminals for the Smart City Environment . IEEE Communications Magazine, 2017, 55 (1), pp.98-106. 10.1109/MCOM.2017.1600225CM . hal-01583705

\section{HAL Id: hal-01583705 \\ https://hal.science/hal-01583705}

Submitted on 8 Sep 2017

HAL is a multi-disciplinary open access archive for the deposit and dissemination of scientific research documents, whether they are published or not. The documents may come from teaching and research institutions in France or abroad, or from public or private research centers.
L'archive ouverte pluridisciplinaire HAL, est destinée au dépôt et à la diffusion de documents scientifiques de niveau recherche, publiés ou non, émanant des établissements d'enseignement et de recherche français ou étrangers, des laboratoires publics ou privés. 


\title{
Efficient media streaming with collaborative terminals for smart city environment
}

\author{
Jordi Mongay Batalla ${ }^{1,2}$, Piotr Krawiec ${ }^{1,2}$, Constandinos X. Mavromoustakis ${ }^{3}$, George \\ Mastorakis $^{4}$, Naveen Chilamkurti ${ }^{5}$, Daniel Négru ${ }^{6}$, Joachim Bruneau-Queyreix ${ }^{6}$ and Eugen \\ Borcoci $^{7}$
}

\author{
${ }^{I}$ National Institute of Telecommunications, Warsaw, Poland \\ ${ }^{2}$ Warsaw University of Technology, Poland \\ ${ }^{3}$ University of Nicosia, Nicosia, Cyprus \\ ${ }^{4}$ Technological Educational Institute of Crete, Heraklion, Crete, Greece \\ ${ }^{5}$ La Trobe University - Melbourne, Australia \\ ${ }^{6}$ LaBRI Lab. - University of Bordeaux, Talence, France \\ ${ }^{7}$ University Politehnica of Bucarest, Bucarest, Romania
}

\begin{abstract}
The efficient provision of multiple services via emerging mobile networking architectures will play a crucial role, towards the realization of the smart city concept. Several of the most demanding (from the required bandwidth point of view) scenarios in smart cities are related with media streaming, which is a key component in smart applications, like Smart Entertainment, Smart Tourism and Smart Surveillance, etc. Such applications are associated with the request of exploiting considerable amount of data, which is difficult to achieve especially in dense urban environments. In this context, this article presents a new solution for HTTP-compliant adaptive media streaming, dedicated to future $5 \mathrm{G}$ mobile networking systems. It aims at increasing bandwidth availability for media streaming, through the use of multiple radio access technologies and direct connections established between devices, if they are in proximity of each-other. The proposed solution considers the scenario, in which a high quality media stream is received by multi-path transmission in the Radio Access Network. The transmission exploits collaboration of neighboring devices, which can use direct device-to-device links. Thus, proxy nodes can be inserted between a given media receiver and access network. Towards ensuring optimized resource allocation at both levels: base-station-to-device and device-to-device, this paper introduces the modules required for collaboration streaming inside Radio Access Networks. The efficiency of the presented system is enhanced through the use of adaptive streaming technology with multiple description coding, well suited to multi-path delivery.
\end{abstract}

\section{Introduction}

Ubiquitous and highly efficient network access service for emerging applications is required for people living in large metropolis. Media content delivery usually need large network resources For example, entertainment or smart tourism applications consume high bandwidth and battery capacity, which are scarce in mobile dense networks. At the present time, video delivery is the 
largest contributor to worldwide traffic in the fixed Internet. Without doubt this service will move to mobile networks as soon as future technologies will be ready. Moreover, multimedia emerging applications will include advanced video definition (4K, $8 \mathrm{~K})$ together with other features as augmented reality, 3D, multi-angle vision, etc. As a result, the multimedia delivery in cities will be extremely demanding in a near future, while wireless network infrastructure is expected to handle much higher traffic volume than today. These requirements should be fulfilled by the next, fifth generation of mobile network $(5 \mathrm{G})$ installed in the access network part all around the city. The underway design of $5 \mathrm{G}$ assumes that the enhancement of the system capacity in urban areas will mainly be achieved by (1) higher spectral efficiency thanks to massive MIMO techniques, (2) infrastructure densification by widespread deployment of small (micro/pico/femto) cells, jointly with support of heterogeneous Radio Access Technologies (RATs), and (3) usage of additional spectrum, particularly from the millimeter-wave region, which may provide high bandwidth, but with relatively short-range coverage due to LOS (Line-of-Sight) propagation with minimal refraction [1].

Additionally to the capacity increase, the Radio Access Network (RAN) in the urban areas should introduce new mechanisms to efficiently manage the RAN resources. Urban area is a specific region characterized by high density, in terms of buildings, population and traffic demand. Consequently, the bandwidth available for users can vary substantially even in neighboring locations. On the other hand, the urban environment increases the probability of a reciprocal close location for several devices and, as a consequence, some of them might have better downlink conditions than others. Therefore, other devices (neighboring a given one, which requested the stream) could be inserted in the media delivery paths, acting as intermediate nodes between media consumer and media source.

This paper proposes a system for collaborative multimedia streaming in smart cities, where the resources control is intelligently managed by the RAN, which governs the network access but also may control the state of the device-to-device (D2D) links. Collaborative streaming is a key step forward for multimedia delivery in physically dense network, as, for example, smart cities.

Some proposals of collaborative streaming have been published before [2,3], but they suffered high response delays because the location of the collaborative streaming management was generally located in overlay systems instead of the RAN [4]. The authors of [5] pointed another challenge in collaborative streaming, which is to find a solution how to attract users to share their resources (downlink bandwidth, battery etc.). Appropriate incentive mechanisms are required, which will result in a win-win situation for all interested parties. This aspect is out of the scope of this paper.

Section 2 presents the RAN architecture for dense heterogeneous networks (e.g., cities) specifying the proposed novel modules and entities responsible for collaborative streaming management. Section 3 shows the enhancements in the end devices required for making the collaborative media streaming feasible. Section 4 discusses the resource allocation in the system, whereas Section 5 presents the requirements for higher layers and, specifically, for codecs and streaming protocols. In this section, we argue that multiple description scheme is necessary for multi-path delivery strategy. At last, the paper is concluded in Section 6.

\section{Access network architecture for collaborative media streaming}

Advanced management procedures and mechanisms are necessary in the cities to manage highly dense HetNets (Heterogeneous Networks). HetNets may increase the efficiency of the radio 
spectrum by dividing the space in micro- and pico-cells. HetNets are based on dense multiple antennas outdoor and indoor. Multiple antennas and multiple RATs may increase the capacity of RAN only if the system is capable of locating each mobile device and executing appropriate algorithms for automatization of the cooperation procedures. Moreover, new mobile architectures (5G) include cooperation of different technologies. At last, the use of cloud resources for control and management of the radio resources could greatly help to the introduction of such complex algorithms.

We propose to increase cooperation of technologies for assuring high-quality multimedia streaming anywhere in the city. Our system assumes that an end user intends to download high bandwidth stream (in general, video) by using HTTP connection. To achieve this objective, a collaborating device (if any is possible) downloads portion of the media, exploiting the downlink bandwidth available for him, and next it transmits the downloaded media to the receiver (end user) via a direct D2D radio connection. High efficiency of such collaborative streaming should involve different wireless technologies with coordinated spectrum usage (for example, LTE for downloading media from the network, and WiFi, Bluetooth or LTE direct to transfer the media directly between user devices).

To assure proper coordination between cells of different levels (macro-, pico-, micro- and D2D connections), an integrated RAN controller is proposed, having access to the set of current network statistics, devices localization and contextual information (including D2D links). The most common architecture of access domain considered in the scope of smart cities, is Cloud-based Radio Access Network (C-RAN, also known as Centralized RAN) [6]. The basic idea of C-RAN is to detach the Baseband Units (BBU), (which are responsible of the signal processing from the remote sites) and move them to a centralized location, whereas the base stations are uniquely equipped with simple Remote Radio Head (RRH) modules (see Figure 1). The RRH task is to perform conversion between digital baseband signals and analog signals transmitted/received by antennas. The BBUs are running as virtualized pool of processing resources in a dedicated data center or using cloud service, and can be shared between different base stations. In this way, the network operators can reduce energy consumption in the mobile infrastructure, decrease both deployment and operational costs of BBU, and also facilitate network upgrades and future migrations to new solutions. Furthermore, emerging Mobile Edge Computing technology enables to expose the spare cloud-computing capabilities available at the RAN to authorized third parties, using open interfaces. It allows to run at the RAN additional functionalities, such as caching and transcoding in case of media streaming. 


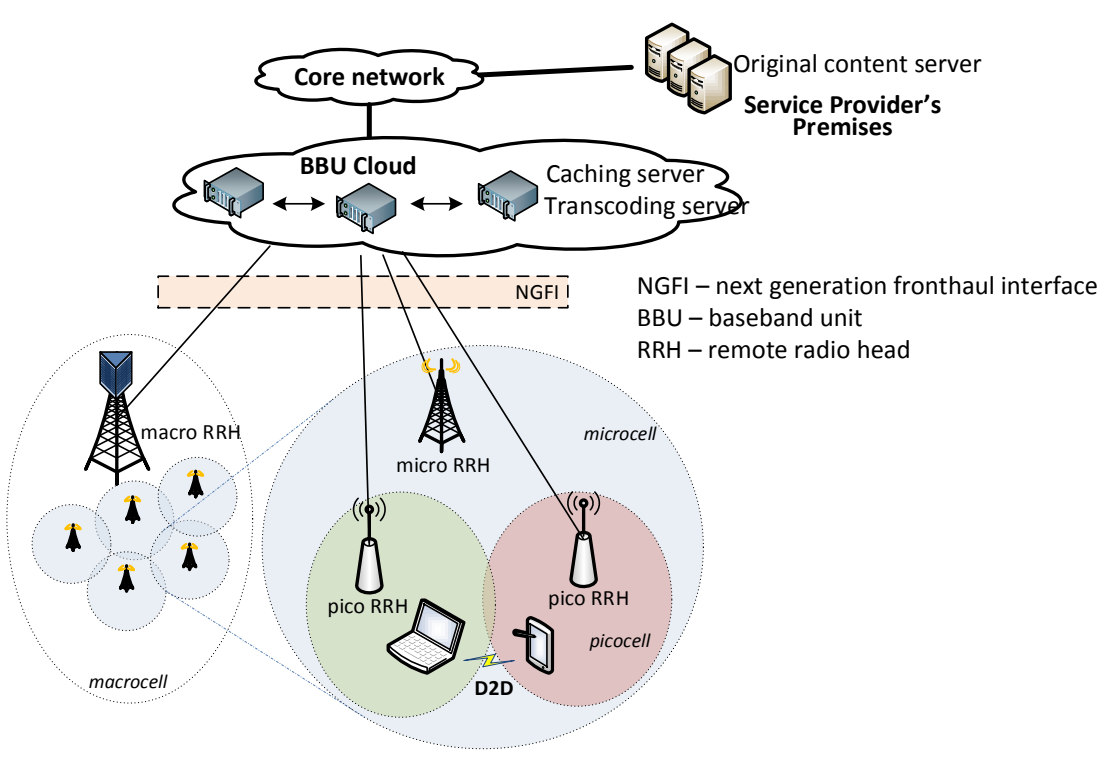

Figure 1. C-RAN architecture.

When deploying collaborative media streaming into $\mathrm{BBU}$, the device contextual information is crucial for taking appropriate decisions about collaboration. Therefore, a centralized BBU architecture is more consistent with presented collaborative media streaming. Although various schemes of splitting the baseband functionality between BBU and RRH are investigated, depending on fronthaul capacity and delay requirements, the most promising is the full-centralized option, when BBU performs baseband processing at three levels of protocol stack, from L1 (i.e. PHY) to L3 layer. It potentially allows for the most efficient use of the radio resources, since the optimization decisions can be based taking into account each layer. Moreover, unlike distributed BBU systems, the optimization executed inside centralized BBU pool can consider multi-RAT potentials. BBU centralization makes cooperation and synchronization of RRHs feasible for sending data to users positioned in close micro-, pico- and macro-cell with the so-called Coordinated Multi-Point (CoMP), which enables better resource utilization due to reduction of inter-cell interferences. The major drawback of fully centralized C-RAN is the highest demands for bandwidth and maximum delay on NGFI (Next Generation Fronthaul Interface), which are met uniquely by fiber links connecting RRHs with BBU pool.

To enable collaborative media streaming, we introduce new modules to the BBU, which aim at providing media content with high quality by exploiting any possible transmission ways available at a given time, even by using D2D connection between neighboring terminals.

The logical architecture of BBU presented in Figure 2 covers modules which perform procedures of the physical and higher layer protocol processing for the supported RAT family. These modules are controlled by Radio resource allocation (RRA) entity. It implements multi-layer (L1 to L3) and multiRAT coordination for attaining high spectral efficiency and throughput, as well as load balancing mechanisms between cells. RRA module can exploit information about terminals physical location, obtained from Localization core, since dense $5 \mathrm{G}$ networks will be able to provide highly accurate device geo-localization [7]. Such information is relevant for managing terminal mobility, because it enables movement tracking and location prediction. 


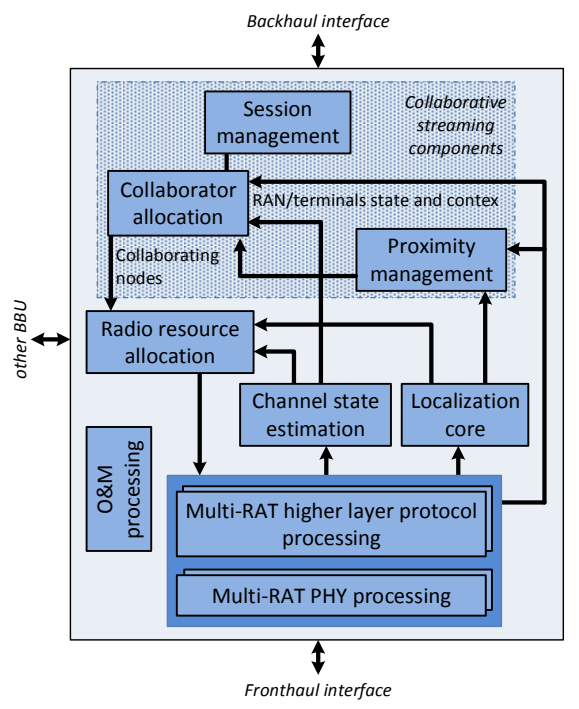

Figure 2. Enhancement of BBU architecture for collaborative media streaming.

The new components added for managing collaborative streaming control the whole process, from finding the appropriate collaborator to assigning data flows to proper path (directly to the destination terminal or via neighboring device). The collaborative streaming control layer in BBU involves Proximity management module that decides which devices/terminals can be considered as neighbors, taking into account not only their physical location, but also the capabilities of the devices as, for example, available wireless interfaces (which can be used to establish direct connections), battery status, etc. Moreover, collaborative streaming components on different BBUs interact to discover terminals which are associated with different base stations, but, at the same time, they are localized at the cell edges and potentially could establish a direct connection between them.

When Proximity management determines the set of potential neighbors, it passes information about them to Collaborator allocation entity. Furthermore, the BBU sends (to collaborating terminals) a command initiating the monitoring of the state of potential D2D connections. Measurement reports from the terminals are collected by Channel state estimation module, which provides (to Collaborator allocation) information about current channel state for each D2D link. Based on this information, Collaborator allocation decides which particular devices, from a set of neighbors, can be used at the current moment as collaborators in media streaming to the given terminal. The module has interface with Radio resource allocation entity to indicate which devices are paired as neighbors and will be used in collaborative streaming.

The module that controls media sessions at the BBU is Session management. It manages video sessions streamed in portions of video upon multiple HTTP requests and ordered by the video content manifest file. Multiple HTTP-based streaming protocols are the most common solutions (e.g., Adobe Dynamic Streaming, Apple's HTTP Live Streaming, Microsoft's ISS Smooth Streaming and Dynamic Adaptive Streaming over HTTP) and their most interesting feature is the capacity of adapting the streaming bitrate to the state of the network. The file ordering all the HTTP request (the fragments of video are independently callable with the own URL) is the manifest file.

The Session management identifies the beginning of a new media session by recognizing the manifest file downloaded by a user. After analyzing the file, Session management is aware of the different fragments of video (called chunks or segments) and the bandwidth necessary for their 
transmission. Next, when the module detects segment request from the user, it asks to Collaborator allocation if additional bandwidth for a given user could be available thanks to collaboration. After checking the potential collaborators and selecting the appropriate one/s, Session management informs to the user about how to request the content fragments of the session through collaborating devices and, concretely, contains the URLs to request the content fragments through different paths (for details about how the content is divided into multi-layer content for multi-path streaming, see Section 5). Let us remark that the content fragments are short in HTTP-based adaptive streaming (2-10 playout seconds), which avoids problems of device mobility due to very short session duration. When a collaborator receives the request through D2D link, it redirects the request to $\mathrm{BBU}$, and the request is further handled by Session management module in BBU.

To speed up media streaming process, Session management works as a caching entity and, based on information provided by the manifest file, it downloads (in advance) a small number of portions of video with best quality (highest bitrate) from the content server. In this way, the module responds to user or collaborators just after receiving media requests, using a priori cached data, to reduce media delivery latency.

\section{Terminal architecture for collaborative applications}

In the proposed solution, process of finding adjacent devices and establishing a direct radio link between them is fully controlled by the BBU. In this way the D2D connection establishment is fast and resource efficient since all required information are provided by the $\mathrm{BBU}$, whereas neighbor discovery process performed by terminals is energy and time-consuming. BBU has the view of the entire access domain, hence it optimizes the selection of neighbors and wireless technology used for D2D connection. Last but not least, network operator performs authentication of collaborating devices, this being important from the point of view of security and privacy. Nonetheless, the set of neighboring devices is restricted only to given operator's clients, what limits the number of potential collaborators. This drawback can be overcome by proper cooperation and collaboration of different network operators (cellular and wireless), intended for forming one ecosystem to cover the smart city area.

Figure 3 presents the high-level architecture of $5 G$ user devices for collaborative streaming, which can play two roles: a collaborator or media receiver. Such devices will be capable of operating in multiple RAT simultaneously. Above L1 and L2 layers components we introduced the entities responsible for collaborative streaming. It includes $D 2 D$ manager, which is a module that establishes and terminates direct link with the neighbor through wireless technology indicated by BBU. Moreover, it manages D2D connections in all the lowest layers. For example, at the MAC layer, the D2D manager sends and receives measurement packets, in appropriate wireless technology, for monitoring direct link conditions. The Monitoring module receives this information and estimates the QoS (Quality of Service) metrics for given direct link, such as estimated bandwidth and packet losses. Measured QoS metrics, as well as contextual information (available wireless interfaces, state of the battery etc.) are transmitted by the Monitoring module to the associated BBU. 

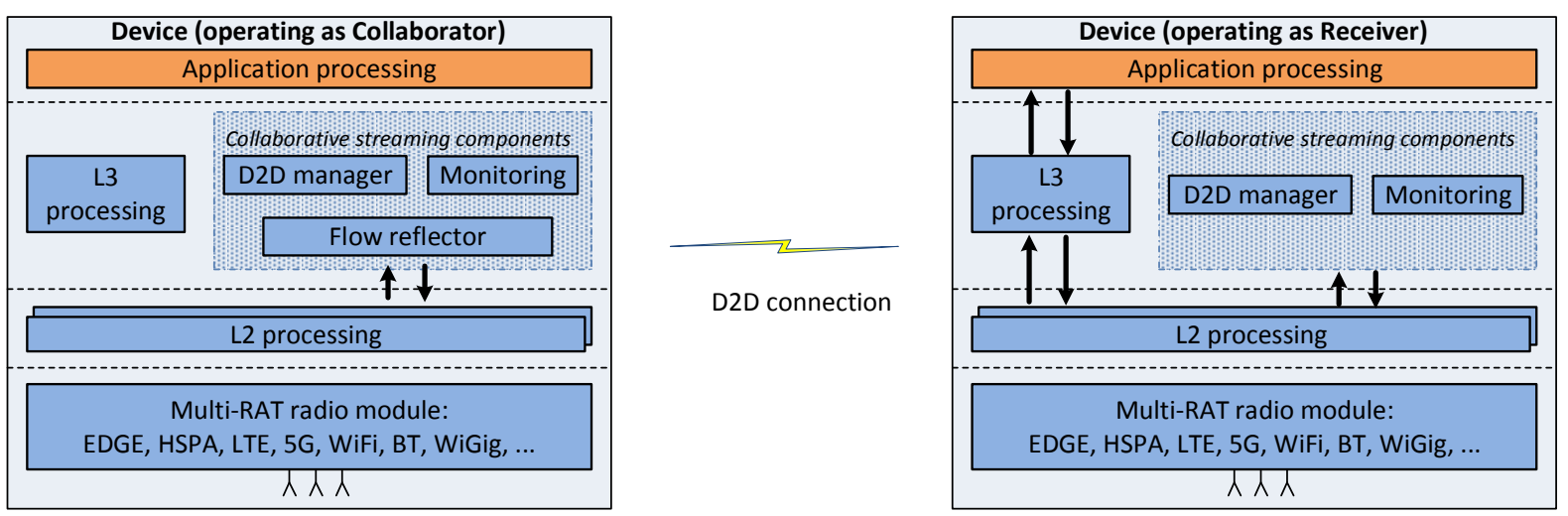

Figure 3. Architecture of client devices for collaborative streaming.

Moreover, a collaborator contains Flow reflector module, which plays the role of a proxy in a communication between the receiver and associated BBU. The module parses the request generated by the receiver, replaces its own address with BBU's address in the request and then transfers it to the BBU. Next, it redirects BBU's response, which carries requested content, to the receiver using D2D connection.

\section{Resource control}

The collaborative streaming is a different scenario comparing to classic D2D approach, where two devices (the source and the destination) are placed in the same access network. On the contrary, collaborative streaming is managed by an entity different to the devices (i.e., the BBU), and the content source is usually in the Internet. Consequently, all involved devices (i.e. the collaborators and the receiver) have to set up connection with their base stations. This is the reason, by which the resource control algorithm for collaborator selection should prefer terminals which allow to establish direct links by wireless technology different from the RAT used in the primary downlink (from base station), to avoid co-channel interferences.

When the BBU selects a set of possible collaborators and sends information about them to the user terminal, the terminal establishes D2D connections with indicated neighbors to measure parameters of the direct links. Measurement results are then returned to BBU. In this way, BBU is aware of the current state of the channel between terminals, and can take it into account during collaborator selection.

In particular, the following input data should be taken into consideration in resource allocation process:

- estimated bandwidth available for each terminal in the downlink (from base station to the terminal),

- RAT supported by terminals,

- estimated bandwidth available between terminals in D2D connection,

- location of terminals,

- prediction of terminal trajectories (to avoid devices which move apart),

- state of the terminal: CPU usage, available energy, wireless interfaces usage,

- activity of the terminal (does any neighbor use a given terminal in another collaborative streaming session?). 
As an output, the resource control algorithm indicates a terminal or terminals, which should be used for collaborative streaming within the scope of given media session. Objectives of the algorithm can vary, according to service providers (if the service provider is inside of the smart city) and/or user requirements and expectations. They may be as follows: to maximize overall media throughput, to maximize throughput while minimize power consumption (in this case, the algorithm should avoid D2D connections which consumes too much energy, even if they provide higher bandwidth), to maximize overall user QoE (the algorithm, for example, should avoid too frequent variation of media streaming bitrate if adaptive streaming technology is used).

In order to ensure high efficiency, the services in smart city environment require cooperation between different actors, including mobile network operators and service providers. Such feature can be achieved through open interfaces provided by Mobile Edge Computing technology. In this way, a service provider will be able to define its own set of input parameters and objectives to be given to the resource control algorithm in order to adapt the service to the application requirement. For example, Smart Entertainment applications require to maximize QoE parameter, whereas Smart Emergency should focus on energy savings to allow longer use of battery powered devices.

The resource control algorithm implemented by Collaborator allocation module is a complex optimization task performed by BBU pool. This is an open research issue that requires further analysis by the investigators; however, an efficient multi-objective algorithm should consider all the parameters and constrains, and should find several solutions belonging to the Pareto frontier instead of one unique solution. Therefore, Evolutionary Multi-objective Optimization [8] seems to be a reasonable approach for solving the tasks of resource control in collaborative streaming in smart cities.

\section{Online content encoding and collaborative media streaming}

Collaborative multimedia delivery is possible in smart cities thanks to high density HetNets, which make the multi-path streaming feasible. Multi-path streaming may be achievable at different levels: from packet level (by sniffing each packet and forwarding through different paths established by overlay routing) to application level (by introducing multiple description coding which allows to encode the content in different and complementary content files). The disadvantage of packet level operations is the high level of processing power necessary for performing deep packet inspection and forwarding operations, which, in practice, disqualifies this option. Also multiple description coding has two important drawbacks: it requires high overhead in terms of bandwidth usage and it is not compatible with the most of video clients in the market.

Therefore, we propose to use a solution provided at the streaming protocol level and called Multiple Description-Dynamic Adaptive Streaming over HTTP (MD-DASH) [9][10]. This protocol is based on well-known Dynamic Adaptive Streaming over HTTTP (DASH), an adaptive multiple HTTP-requests streaming protocol that quickly reaches the majority position in the market thanks to the fact that it is an open standard. In DASH standard, the original video content is divided in different chunks (called segments) and encoded in multiple bitrates (all the content segments encoded with the same nominal bitrate belong to the same representation). The end user requests each segment independently (new HTTP request) and selects the best representation that may be streamed for each requested segment by analyzing the situation of the network. 
The solution presented in [9][10] (MD-DASH) makes multi-path streaming feasible at the HTTP level (each HTTP request is served through a different path), avoiding operations at the packet level. Moreover, MD-DASH is easily implementable in current applications (since only the adaptation protocol library should be extended) and does not require excessive overhead.

The idea of MD-DASH is to interleave groups of pictures (GOP) that belongs to higher and lower representations creating a sub-stream, whose mean bitrate is between lower and higher representative bitrates. Each sub-stream is fully decodable and viewable but the playing-out of one unique sub-stream offers sub-optimal Quality of Experience ( $Q \circ E)$ to the viewer since some parts of the content will be played with higher quality than others. The play-out of all different sub-streams together offers optimal QoE due to the capacity of the MD-DASH client to select from each substream the best quality GOPs discarding the lower quality ones.

The GOPs are encoding operations units used in current (H.264) and upcoming (H.265) codecs. One GOP contains a video of the same scene, such that the encoding operations inside one GOP are highly correlated, which improves the possibility to compression ratio. Figure 4 shows MD-DASH encoding based on two different representations for three sub-streams, each one transmitted through a different path within the network.

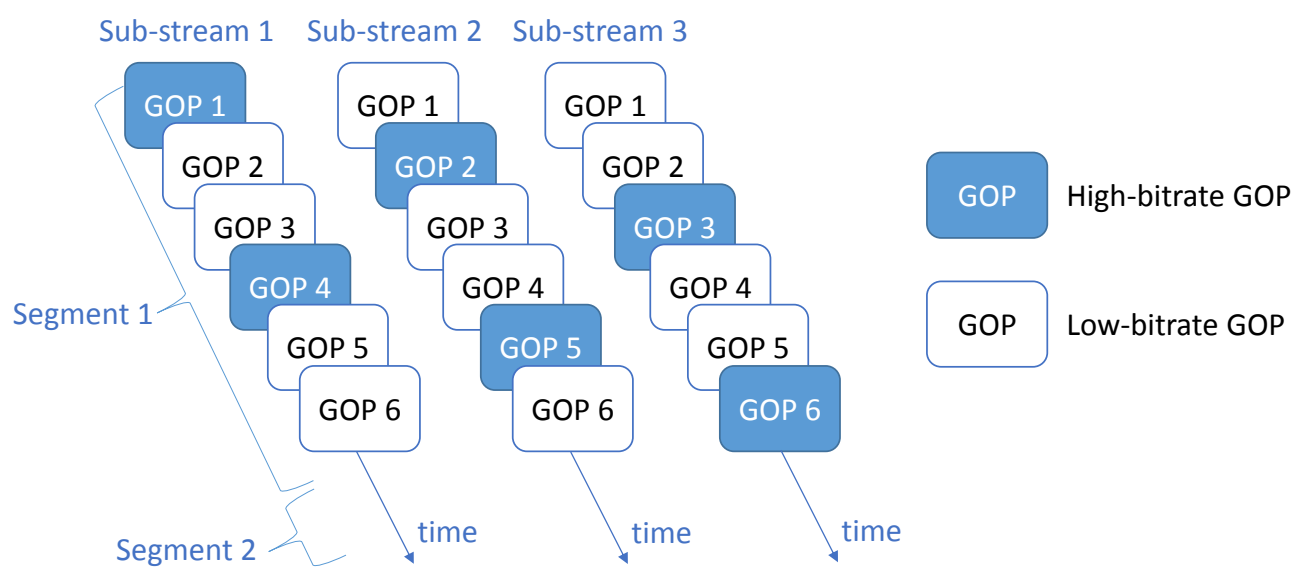

Figure 4. MD-DASH scheme. Encoding strategy

The High-bitrate GOPs contain high-definition information of the video, whereas low-bitrate GOPs contain the minimum video information that is necessary for playing the video with lowest quality. Therefore, the overhead introduced by MD-DASH is low (and equals the relation between low- and high-bitrate GOPs, as indicated in [9] [10]). The end user decides about the representation to be downloaded in each segment (say segment $i$ ), and initiates the HTTP session with the BBU.

The transcoding server functionality in the BBU cloud is in charge of preparing the appropriate substreams for segment $i$ taking into account the number of available collaborators (say $N$ ) and the available bandwidth on each D2D link, in such a way that the high-bitrate GOPs are encoded with the representation bitrate indicated by the end user. In the case that all the $N$ paths have similar downlink capacity, the $N$ sub-streams will contain a similar number of high-bitrate GOPs. Whereas, in the case that the $N$ paths have different bottleneck bitrates, the sub-stream for each path will be adapted to the path bottleneck. For this scope, the transcoding server is instructed by the BBU Collaborator allocation module about the bitrate of each path. The original content stored in the 
BBU transcoding server is downloaded from the original content server (at the service provider's premises) with the highest representation, so that the BBU may transcode to any of the lowest representations, as required by the end user.

We evaluated the MD-DASH proposal by considering the perceived quality at the consumer's side, i.e., QoE. To this end, we monitored and evaluated 2 criterias considered as essential for the QoE of video streaming services: the number of rebuffering events, and the quality distribution throughout the streaming session. Our benchmark includes three implemented streaming applications based on dash.js player [2]: one MDC-streaming client (3 servers simultaneously, no adaptation features, requesting servers with segments containing $33 \%$ of GOPs at $6 \mathrm{Mbps}$ and $66 \%$ of GOPs at $200 \mathrm{Kbps}$ ), one MD-DASH client (including bitrate adaptation and server switching mechanism, using 3 servers simultaneously and being able to switch to 6 other available servers), and one DASH-ORACLE application (using one server at a time, with 3 available servers) being aware of upcoming bandwidth fluctuations and knowing exactly when and how to perform adaptation and server switching. quality (defined as the overhead from description creation). The 10-minute Big Buck Bunny movie was made available at the transcoding server side at 7 different bitrates in H.264 (200Kbps, 1Mbps, 1.5Mbps, $2 \mathrm{Mbps}, 3 \mathrm{Mbps}, 4 \mathrm{Mbps}$ and $6 \mathrm{Mbps}$ ) and segments were composed of $12 \mathrm{GOPs}$ each. The MD-based clients are selecting the $200 \mathrm{Kbps}$ quality as the redundancy to be inserted into requested segments in order to minimize the overhead.

The DASH Industry Forum provides benchmarks for various aspects of the DASH standard [DASH]. The benchmarks include twelve different network profiles (NPs) featuring different bandwidths, delays and packet loss. Each profile spends 30 seconds for each step described in Table 1, then starts back at the beginning. For each experiment, a specific NP is associated to all channels between the client and the servers. A random time offset is set to each assigned NP to represent bandwidth diversity and variability in the network. Each video was repeated 40 times per application and per NP and a total playback time of 120 hours was performed.

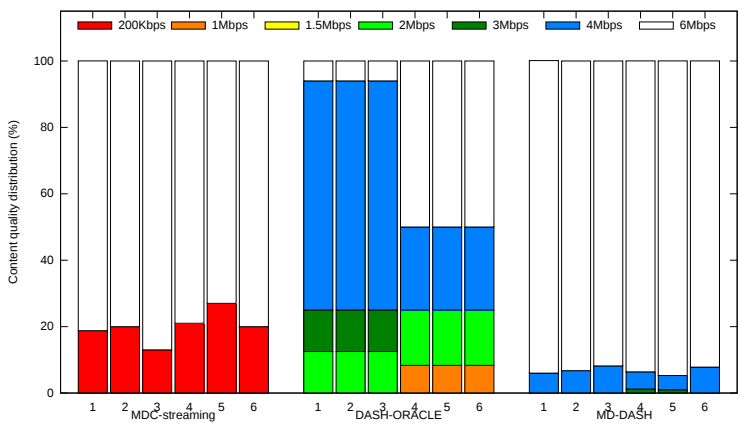

Figure 5: Quality representation throughout streaming session

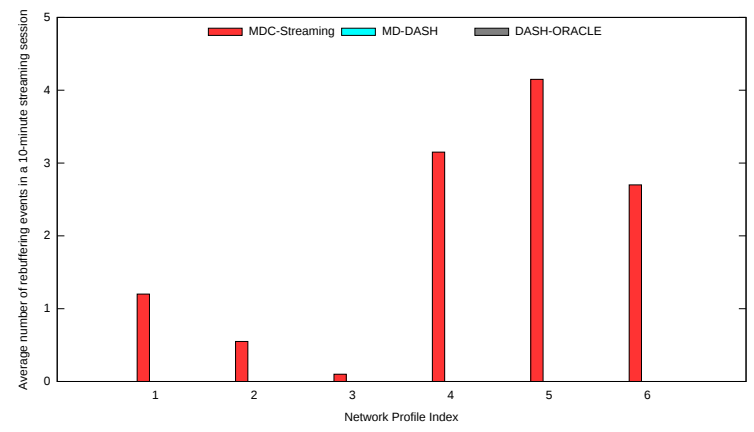

Figure 6: Average number of rebuffering events

Table 1: Network Profiles

\begin{tabular}{|c|l|l|l|l|l|}
\hline $\begin{array}{c}1 \\
\text { Mbps (ms,\%) }\end{array}$ & \multicolumn{1}{|c|}{$\begin{array}{c}2 \\
\text { Mbps (ms,\%) }\end{array}$} & $\begin{array}{c}3 \\
\text { Mbps (ms,\%) }\end{array}$ & $\begin{array}{c}4 \\
\text { Mbps (ms,\%) }\end{array}$ & $\begin{array}{c}5 \\
\text { Mbps (ms,\%) }\end{array}$ & $\begin{array}{c}6 \\
\text { Mbps (ms,\%) }\end{array}$ \\
\hline $5.0(38 ; 0.09)$ & $5.0(13 ; 0.81)$ & $5.0(11 ; 1.00)$ & & & \\
$4.0(50 ; 0.08)$ & $4.0(18 ; 0.63)$ & $4.0(13 ; 1.25)$ & $9.0(25 ; 0.06)$ & $9.0(10 ; 0.40)$ & $9.0(6 ; 1.00)$ \\
$3.0(75 ; 0.06)$ & $3.0(28 ; 0.44)$ & $3.0(15 ; 1.50)$ & $4.0(50 ; 0.07)$ & $4.0(50 ; 0.08)$ & $4.0(13 ; 1.25)$ \\
$2.0(88 ; 0.09)$ & $2.0(58 ; 0.21)$ & $2.0(20 ; 1.75)$ & $2.0(75 ; 0.10)$ & $2.0(150 ; 0.03)$ & $2.0(20 ; 1.50)$ \\
$1.5(100 ; 0.12)$ & $1.5(200 ; 0.03)$ & $1.5(25 ; 2.00)$ & $1.0(100 ; 0.16)$ & $1.0(200 ; 0.07)$ & $1.0(25 ; 2.00)$ \\
$2.0(88 ; 0.09)$ & $2.0(58 ; 0.21)$ & $2.0(20 ; 1.75)$ & $2.0(75 ; 0.10)$ & $2.0(150 ; 0.03)$ & $2.0(20 ; 1.50)$ \\
$3.0(75 ; 0.06)$ & $3.0(28 ; 0.44)$ & $3.0(15 ; 1.50)$ & $4.0(50 ; 0.07)$ & $4.0(50 ; 0.08)$ & $4.0(13 ; 1.25)$ \\
$4.0(50 ; 0.08)$ & $4.0(18 ; 0.63)$ & $4.0(13 ; 1.25)$ & & & \\
\hline
\end{tabular}


MDC-Streaming displayed the top content quality for approximately $78 \%$ of the time on all NPs (see Fig. (ref\{fig:percent1\}). MD-DASH performed a trade-off between content quality and network availability. Beside, the server-switching mechanisms in MD-DASH allowed the client to take benefit of greater link diversity and eventually provided the $6 \mathrm{Mbps}$ top quality for $84 \%$ of the time in average on all NPs. In terms of quality distribution over the streaming session, MD-DASH performed significantly better than the DASH-ORACLE, thanks to the simultaneous usage of servers.

For the MDC-Streaming client, rebuffering occured between 0.12 and 1.22 times per streaming session on NPs 1-2-3 and between 2.76 and 4.08 times per session on NPs 4-5-6. Thanks to its bitrate adaptation and server switching mechanism, MD-DASH avoided buffer depletion on all NPs. With DASH-ORACLE, rebuffering never took place. These results demonstrate the asset of this protocol in terms of QoE for online content encoding and for collaborative media streaming for the proposed CRAN architecture.

\section{Conclusions}

This article presents a solution for growing media delivery throughput in dense heterogeneous wireless networks. It is based on collaboration of the terminals in proximity of each other, for providing multi-path delivery of media segments. The multi-path transmission is well suited to increase efficiency in future $5 G$ systems, which respond to smart city requirements for communication infrastructure. We described new modules designated for user devices as well as centralized RAN to allow collaboration. At the client side, such modules are responsible for establishing and managing D2D links. They also implement mechanisms of relaying data between media receiver and the network. On the other hand, modules introduced to the BBU are responsible for selecting appropriate collaborators and optimal resource allocation. In the article, we discussed a set of parameters and major challenges to be addressed by the resource allocation algorithm.

Multi-path delivery in C-RAN enables to use novel MD-DASH streaming technology, which assumes generating a number of sub-streams transferred to the receiver through different paths. The more sub-streams reach user's terminal, the higher QoE will be perceived by the user during media consumption. In the presented system process of sub-stream creation is performed using computing capabilities provided by the C-RAN, in accordance with the instructions from the BBU resource manager. Such application-aware approach allows to fit content characteristics to the current C-RAN conditions.

\section{References}

[1] S. Talwar, D. Choudhury, K. Dimou, E. Aryafar, B. Bangerter, K. Stewart, "Enabling Technologies and Architectures for $5 \mathrm{G}$ Wireless". Microwave Symposium (IMS), IEEE MTT-S International. Tampa (FL), US (2014)

[2] M. Zhong, H. Peizhao, J. Indulska, M.J. Kumar, "ColStream: Collaborative streaming of ondemand videos for mobile devices", In Proc. of IEEE 15th International Symposium on a World of Wireless, Mobile and Multimedia Networks, Sydney, Australia (2014)

[3] N. Narendra, P.K. Reddy, K. Kumar, A. Varghese, P. Swamy, G. Chandra, P. Balamuralidhar, "MobiCoStream: Real-time collaborative video upstream for Mobile Augmented Reality 
applications", in Proc. of 2014 IEEE International Conference on Advanced Networks and Telecommuncations Systems (ANTS), (2014)

[4] E. Toledano, D. Sawada, A. Lippman, H. Holtzman, F. Casalegno, "CoCam: A collaborative content sharing framework based on opportunistic P2P networking", in Proc. of the Consumer Communications and Networking Conference (CCNC), Las Vegas, US (2013)

[5] X. Changqiao, J. Shijie, M. Wang, L. Zhong, H. Zhang, G.M. Muntean, "Performance-Aware Mobile Community-Based VoD Streaming Over Vehicular Ad Hoc Networks", IEEE Transactions on Vehicular Technology, Volume 64, Issue 3, pp. 1201 - 1217, (2015)

[6] A. Checko, H.L. Christiansen, Y. Yan, L. Scolari, G. Kardaras, M.S. Berger, L. Dittmann, "Cloud RAN for Mobile Networks - a Technology Overview". IEEE Communications Surveys \& Tutorials, Volume:17, Issue: 1, Pp.: 405 - 426. (2015)

[7] A. Hakkarainen, J. Werner, M. Costa, K. Leppanen, M. Valkama, "High-Efficiency Device Localization in 5G Ultra-Dense Networks: Prospects and Enabling Technologies". IEEE 82nd Vehicular Technology Conference (VTC Fall), (2015)

[8] N. Padhye, L. Zuo, C.K. Mohan and P.K. Varshney: "Dynamic and Evolutionary Multi-objective Optimization for Sensor Selection in Sensor Networks for Target Tracking", Proceedings of the International Joint Conference on Computational Intelligence, Funchal, Portugal (2009)

[9] J. Bruneau-Queyreix, D. Négru, J. Mongay Batalla, E. Borcoci, "Multiple Description-DASH: Pragmatic video streaming maximizing End-Users' Quality of Experience". To be published in IEEE International Conference on Communications ICC. Kuala Lumpur, Malaysia, (2016)

[10] J. Bruneau-Queyreix, M. Lacaud, D. Négru, J. Mongay Batalla, E. Borcoci, "MS-Stream: A Multiple-Source Adaptive Streaming Solution Enhancing Consumer's Perceived Quality," IEEE Consumer Communications and Networking Conference - CCNC, 2017. 